M. OZAWA

KODAI MATH. J.

6 (1983), 261-277

\title{
ON A NEW CLASS OF ULTRAHYPERELLIPTIC SURFACES
}

\author{
By Mitsuru Ozawa
}

1. Introduction. Let $R$ be an ultrahyperelliptic surface defined by $y^{2}=g(x)$ with an entire function $g(x)$ having only an infinite number of simple zeros. Let $\mathscr{M}(R)$ be the class of non-constant meromorphic functions on $R$. Let $P(f)$ be the number of lacunary values of $f$ in $\mathscr{M}(R)$. Let $P(R)$ be $\sup _{f \in \mathscr{M}(R)} P(f)$. This quantity is called the Picard constant of $R$. In the ultrahypere lliptic case $2 \leqq$ $P(R) \leqq 4$. Surfaces with $P(R)=2$ or 4 are completely determined and those with $P(R)=3$ are still undetermined except for those of finite order [2], [8]. Let $S$ be another ultrahyperelliptic surface defined by $Y^{2}=G(X)$ with a similar entire function $G(X)$. Let $\phi$ be a non-trivial analytic mapping of $R$ into $S$. Then $P(R) \geqq P(S)$. The existence of $\phi$ is equivalent to the existence of entire $h$ and meromorphic $f$ satisfying

$$
f(z)^{2} g(z)=G(h(z)) .
$$

Here $h$ is called the projection of $\phi$ and is defined by

$$
S \circ \phi \circ \mathscr{Q}_{R}^{-1}
$$

with $\mathscr{P}_{R}:(x, y) \rightarrow x$ and $\mathscr{P}_{S}:(X, Y) \rightarrow X$. This $h$ is one-valued which is equivalent to the rigidity of $\phi[6],[7]$. The above functional equation gives a powerful tool to get several criteria for the existence of analytic mappings [1], [2], [7], [8], [9], [10].

In this paper we shall introduce a new class of surfaces. Let $R$ be an ultrahyperelliptic surface defined by $y^{2}=g(x)$ with entire $g(x)$ having only an infinite number of simple zeros. Let $\mathcal{E}(R)$ be the set of non-constant regular function on $R$. If there is a member $f$ in $\mathcal{E}(R)$ satisfying the following conditions, then $R$ is called of maximal $B$ type:

(1) There are constants $a \neq \infty, c \neq 0$ satisfying

$$
a^{2}-2 a f_{1}+f_{1}^{2}-f_{2}^{2} g=c
$$

when $f \circ \mathcal{Q}_{R}^{-1}(x)$ is represented as $f_{1}(x)+f_{2}(x) \sqrt{g(x)}$.

(2) There are systems $\left(a_{1}, \cdots, a_{t}\right)$ and $\left(n_{1}, \cdots, n_{t}\right)$ such that for each $j$ all the roots of $f=a$, have their orders $n_{j} p_{j k}$ with integers $n_{j} \geqq 2$ and $p_{j k} \geqq 1$. Further $\left(n_{1}, \cdots, n_{t}\right)$ satisfies

Received January 10, 1983 


$$
\sum_{j=1}^{t}\left(1-\frac{1}{n_{\jmath}}\right)=2
$$

We shall decide the surfaces of maximal $B$ type and discuss the existence problem of analytic mappings.

2. In order to go further we need several preparations. We firstly remark that

$$
\sum_{j=1}^{t}\left(1-\frac{1}{n_{\jmath}}\right) \leqq 2
$$

in general. The Nevanlinna-Selberg theory [11] of two-valued algebroid functions gives

$$
(q-4) T(r, f)<\sum_{1}^{q} N\left(r, w_{\nu}\right)--N\left(r, W_{f}\right)+O(\log r T(r, f)) .
$$

Our function $f$ satisfies $N(r, \infty)=0, N(r, a)=0$. Further

$$
N\left(r, W_{f}\right) \geqq \sum_{\substack{f \\ f^{\prime}\left(z_{0}\right) \neq \infty \\\left(z_{0}\right) \neq \infty}}\left\{m\left(z_{0}\right)-1\right\}
$$

with the multiplicity $m\left(z_{0}\right)$ at $z_{0}$. Hence

$$
(q-4) T(r, f)<\sum_{1}^{q-2} \bar{N}\left(r, w_{\nu}\right)+O(\log r T(r, f)), \quad w_{\nu} \neq \infty, a .
$$

Now we put $w_{\nu}=a_{\nu}, q-2=t$. Then

$$
(t-2) T(r, f)<\sum_{\jmath=1}^{t} \frac{1}{n_{\jmath}} N\left(r, a_{\jmath}\right)+O(\log r T(r, f)) .
$$

Hence

$$
\sum_{\jmath=1}^{t}\left(1-\frac{1}{n_{\jmath}}\right) \leqq 2
$$

Recently Toda [12] had proved the following fact:

Let $f_{0}, \cdots, f_{p}(p \geqq 1)$ be $p+1$ non-constant entire functions and let $a_{0}, \cdots, a_{p}$ be $p+1$ meromorphic functions $(\not \equiv)$ in $|z|<\infty$ such that $T\left(r, a_{j}\right)=o\left(T\left(r, f_{j}\right)\right)$, $\jmath=0, \cdots, p$. Then, if

$$
\sum_{j=0}^{p} a_{j}(z) f_{j}^{n_{j}}(z)=1
$$

for some integers $n_{0}, \cdots, n_{p}(\geqq 1)$,

$$
\sum_{j=0}^{p} \frac{1}{n_{\jmath}} \geqq \frac{1}{p}
$$

3. In this section we shall decide all the surfaces of maximal $B$ type. 
Firstly we may assume that $a=0$ and hence $a_{\jmath} \neq 0$. by

$$
\sum_{\jmath=1}^{t}\left(1-\frac{1}{n_{\jmath}}\right)=2
$$

we have the following four possibilities:

i) $t=3, \quad n_{1}=2, \quad n_{2}=3, \quad n_{3}=6$;

ii) $t=3, \quad n_{1}=2, \quad n_{2}=4, \quad n_{3}=4$;

iii) $t=3, \quad n_{1}=3, \quad n_{2}=3, \quad n_{3}=3$;

iv) $t=4, \quad n_{1}=2, \quad n_{2}=2, \quad n_{3}=2, \quad n_{4}=2$.

Case i). Let us consider the two-valued entire algebroid function satisfying

$$
F(z, y) \equiv y^{2}+2 A y+c=0 .
$$

Then $F(z, 0)=c$. Further with entire $g_{1}, g_{2}, g_{3}$

Hence

$$
\begin{aligned}
& F\left(z, a_{1}\right)=g_{1}^{2}, \\
& F\left(z, a_{2}\right)=g_{2}^{3}, \\
& F\left(z, a_{3}\right)=g_{3}^{6} .
\end{aligned}
$$

$$
a_{3} g_{2}^{3}-a_{2} g_{3}^{6}=\left(a_{3}-a_{2}\right)\left(c-a_{2} a_{3}\right) .
$$

If $c \neq a_{2} a_{3}$, then Toda's result gives a contradiction. If $c=a_{2} a_{3}$, then $a_{2} g_{3}^{6}=a_{3} g_{2}^{3}$. This shows that $g_{2}$ has only zeros of even order $\geqq 2$, that is, $g_{2}$ can be written as $g_{4}^{2}$. Hence we may put $n_{2}=6$, which is a contradiction by

$$
\sum_{\jmath=1}^{3}\left(1-\frac{1}{n_{\jmath}}\right)=\frac{13}{6}>2 \text {. }
$$

When $g_{2}$ has no zero, then we may put $n_{2}=\infty$ and $n_{3}=\infty$. This is again impossible.

Case ii). This case is impossible by the similar reasoning as in case i).

Case iii). In this case

$$
F\left(z, a_{j}\right)=g_{\jmath}^{3}, \quad \jmath=1,2,3 .
$$

Hence

$$
a_{1} g_{2}^{3}-a_{2} g_{1}^{3}=\left(a_{1}-a_{2}\right)\left(c-a_{1} a_{2}\right) .
$$

If $c \neq a_{1} a_{2}$, then this is impossible by Toda's result. If $c=a_{1} a_{2}$, then $c \neq a_{1} a_{3}$ and hence

$$
a_{1} g_{3}^{3}-a_{3} g_{1}^{3}=\left(a_{1}-a_{3}\right)\left(c-a_{1} a_{3}\right)
$$

implies a contradiction.

Case iv). In this case

$$
F\left(z, a_{j}\right)=g_{\jmath}^{2}, \quad \jmath=1,2,3,4 .
$$


We firstly prove that $c \neq a_{1} a_{2}, c \neq a_{2} a_{3}$ implies $c=a_{1} a_{3}$. By $c \neq a_{1} a_{2}, c \neq a_{2} a_{3}$ we have

$$
\begin{aligned}
& \gamma_{2}^{2} g_{2}^{2}-\gamma_{1}^{2} g_{1}^{2}=1, \quad \gamma_{2}^{2}=\frac{a_{1}}{\left(a_{1}-a_{2}\right)\left(c-a_{1} a_{2}\right)}, \quad \gamma_{1}^{2}=\frac{a_{2}}{\left(a_{1}-a_{2}\right)\left(c-a_{1} a_{2}\right)}, \\
& \gamma_{3}^{2} g_{3}^{2}-\gamma_{2}^{* 2} g_{2}^{2}=1, \quad \gamma_{3}^{2}=\frac{a_{2}}{\left(a_{2}-a_{3}\right)\left(c-a_{2} a_{3}\right)}, \quad \gamma_{2}^{* 2}=\frac{a_{3}}{\left(a_{2}-a_{3}\right)\left(c-a_{2} a_{3}\right)} .
\end{aligned}
$$

Hence

$$
\begin{aligned}
& \gamma_{2} g_{2}-\gamma_{1} g_{1}=\beta_{1} e^{H_{1}}, \\
& \gamma_{2} g_{2}+\gamma_{1} g_{1}=\frac{1}{\beta_{1}} e^{-H_{1}}
\end{aligned}
$$

with entire $H_{1}, H_{1}(0)=0$ and a constant $\beta_{1} \neq 0$. Thus

$$
\gamma_{2} g_{2}=\frac{1}{2}\left(\beta_{1} e^{H_{1}}+\frac{1}{\beta_{1}} e^{-H_{1}}\right) .
$$

Similarly we have

$$
\gamma_{2}^{*} g_{2}=\frac{1}{2}\left(\frac{1}{\beta_{2}} e^{-H_{2}}-\beta_{2} e^{H_{2}}\right)
$$

with entire $H_{2}, H_{2}(0)=0$ and a non-zero constant $\beta_{2}$. Hence

$$
\gamma_{2}^{*} \beta_{1} d^{H_{1}}+\frac{\gamma_{2}^{*}}{\beta_{1}} e^{-H_{1}}=\frac{\gamma_{2}}{\beta_{2}} e^{-H_{2}}-\beta_{2} \gamma_{2} e^{H_{2}}
$$

By the impossibility of Borel's identity we have two possibilities

$$
\left\{\begin{array} { l } 
{ H _ { 2 } = H _ { 1 } } \\
{ \gamma _ { 2 } ^ { * } \beta _ { 1 } = - \beta _ { 2 } \gamma _ { 2 } } \\
{ \gamma _ { 2 } ^ { * } \beta _ { 2 } = \beta _ { 1 } \gamma _ { 2 } , }
\end{array} \quad \left\{\begin{array}{l}
H_{2}=-H_{1} \\
\gamma_{2}^{*} \beta_{1} \beta_{2}=\gamma_{2} \\
\gamma_{2}^{*}=-\beta_{1} \beta_{2} \gamma_{2} .
\end{array}\right.\right.
$$

In both cases we have

$$
\gamma_{2}^{* 2}+\gamma_{2}^{2}=0
$$

which gives $c=a_{1} a_{3}$.

The above fact gives the following possibilities:

$$
\left(\begin{array} { l } 
{ c = a _ { 1 } a _ { 2 } } \\
{ c = a _ { 3 } a _ { 4 } , }
\end{array} \quad \left(\begin{array}{l}
c=a_{1} a_{3} \\
c=a_{2} a_{4},
\end{array}, \quad\left(\begin{array}{l}
c=a_{1} a_{4} \\
c=a_{2} a_{3} .
\end{array}\right.\right.\right.
$$

We may restrict to the first case. Hence

Since $c \neq a_{2} a_{3}$

$$
a_{1} g_{2}^{2}=a_{2} g_{1}^{2}, \quad a_{4} g_{3}^{2}=a_{3} g_{4}^{2} \text {. }
$$

$$
\gamma_{3} g_{3}=\frac{1}{2}\left(\frac{1}{\beta} e^{-H}-\beta e^{H}\right)
$$


with entire $H, H(0)=0$ and a constant $\beta \neq 0$ and

$$
\gamma_{3}^{2}=\frac{1}{\left(a_{3}-a_{2}\right)\left(a_{1}-a_{3}\right)} \text {. }
$$

Hence

$$
\begin{aligned}
& A=\frac{1}{2 a_{3}}\left\{\frac{1}{4 \gamma_{3}^{2}}\left(\beta e^{H}-\frac{1}{\beta e^{H}}\right)^{2}-a_{1} a_{2}-a_{3}\right\} \\
& C-A^{2}=-\frac{1}{64 a_{3}^{2} \gamma_{3}^{4}}\left\{\left(\beta e^{H}-\frac{1}{\beta e^{H}}\right)^{4}-2 u\left(\beta e^{H}-\frac{1}{\beta e^{H}}\right)^{2}+v^{2}\right\}
\end{aligned}
$$

with

$$
u=\frac{4\left(a_{1} a_{2}+a_{3}^{2}\right)}{\left(a_{3}-a_{2}\right)\left(a_{1}-a_{2}\right)}, \quad v^{2}=-\frac{16\left(a_{3}^{2}-a_{1} a_{2}\right)^{2}}{\left(a_{3}-a_{2}\right)^{2}\left(a_{1}-a_{2}\right)^{2}} .
$$

Further with a constant $K$

$$
C-A^{2}=K\left\{\left(\beta e^{H}-\frac{1}{\beta e^{H}}\right)^{2}-\delta_{1}\right\}\left\{\left(\beta e^{H}-\frac{1}{\beta e^{H}}\right)^{2}-\delta_{2}\right\} .
$$

Here $\delta_{1} \delta_{2}\left(\delta_{1}-\delta_{2}\right) \neq 0$ and $\left(\delta_{1}+4\right)\left(\delta_{2}+4\right) \neq 0 . \quad$ In fact $\delta_{1}=0$ gives $u^{2}=u^{2}-v^{2}, v=0$, that is, $a_{3}^{2}=a_{1} a_{2}$. Hence $a_{1} a_{2}=a_{3} a_{4}$ gives $a_{2}=a_{4}\left(a_{3} \neq 0\right)$. This is impossible. If $\delta_{1}=\delta_{2}, u^{2}=v^{2}$ and hence $a_{3}=0$ or $a_{1} a_{2}=0$, which is again impossible. $\delta_{1}=-4$ implies $16+8 u+v^{2}=0$. This gives $a_{3}=0$ or $a_{1}=a_{2}$, which is impossible.

We may write

$$
C-A^{2}=\frac{K}{\beta^{4} e^{4 H}} \prod_{j=1}^{8}\left(\beta e^{H}-\lambda_{\jmath}\right) .
$$

Hence

$$
\begin{aligned}
& \lambda_{1} \lambda_{2}=\lambda_{3} \lambda_{4}=\lambda_{5} \lambda_{6}=\lambda_{7} \lambda_{8}=-1, \\
& \lambda_{1}+\lambda_{2}=-\lambda_{3}-\lambda_{4}=-\sqrt{\delta_{1}}, \quad \lambda_{5}+\lambda_{6}=-\lambda_{7}-\lambda_{8}=-\sqrt{\delta_{2} .}
\end{aligned}
$$

$\delta_{1} \neq-4, \quad \delta_{2} \neq-4$ imply $\lambda_{1} \neq \lambda_{2}, \quad \lambda_{3} \neq \lambda_{4}, \quad \lambda_{5} \neq \lambda_{6}, \quad \lambda_{7} \neq \lambda_{8} . \quad$ Further $\lambda_{2} \neq \lambda_{\jmath}$ if $i \neq \jmath$ and $\lambda_{j} \neq 0$.

LEMma. Let $N_{1}\left(r, \gamma, e^{H}\right)$ be the counting function of multiple zeros of $e^{H}-\gamma$, $\gamma \neq 0$. Then

$$
N_{1}\left(r, \gamma, e^{H}\right)=o\left(m\left(r, e^{H}\right)\right) .
$$

Let $N_{2}\left(r, \gamma, e^{H}\right)$ be the counting function of simple zeros of $e^{H}-\gamma, \gamma \neq 0$. Then

$$
N_{2}\left(r, \gamma, e^{H}\right) \sim m\left(r, e^{H}\right) .
$$

This was proved in [5]. It is evident that $e^{H}-\lambda_{1}=0, e^{H}-\lambda_{2}=0$ have no common root if $\lambda_{1} \neq \lambda_{2}, \lambda_{1} \lambda_{2} \neq 0$. These facts imply that $C-A^{2}$ has infinitely many simple zeros.

Since every $f$ in $\mathcal{E}(R)$ can be represented as 
$f$ satisfies

$$
-A+f_{2} \sqrt{g},
$$

Hence

$$
y^{2}+2 A y+A^{2}-f_{2}^{2} g=0 \text {. }
$$

Let us put

$$
-f_{2}^{2} g=c-A^{2} .
$$

$$
\beta e^{H}-\lambda_{\jmath}=m_{j}(z)^{2} L_{j}(z),
$$

where $L$, has only simple zeros. Then

$$
c-A^{2}=-\frac{K}{\beta^{4} e^{4 H}} \prod_{\jmath=1}^{8} m_{\jmath}(z)^{2} \prod_{j=1}^{8} L_{j}(z) .
$$

Hence we may put

$$
g=\prod_{j=1}^{8} L_{j}(z) .
$$

However there does not occur any trouble even if we adopt

$$
\prod_{j=1}^{8}\left(\beta e^{H}-\lambda_{j}\right), \quad H(0)=0
$$

as $g$, since the structure of $R$ is invariant under this change and $\mathcal{E}(R)$ is too. Hence we put

$$
g=\prod_{\jmath=1}^{8}\left(\beta e^{H}-\lambda_{j}\right), \quad H(0)=0 .
$$

Here $\lambda_{\imath} \neq \lambda_{\jmath}$ for $\imath \neq \jmath, \lambda_{\jmath} \neq 0$ and further

$$
\begin{aligned}
& \lambda_{1} \lambda_{2}=\lambda_{3} \lambda_{4}=\lambda_{5} \lambda_{6}=\lambda_{7} \lambda_{8}=-1, \\
& \lambda_{1}+\lambda_{2}=-\lambda_{3}-\lambda_{4}=-\sqrt{\delta_{1}}, \quad \lambda_{5}+\lambda_{6}=-\lambda_{7}-\lambda_{8}=-\sqrt{\delta_{2}} .
\end{aligned}
$$

Another representation of $g$ is

$$
g=\beta^{8} e^{8 H}-A_{2} \beta^{6} e^{6 H}+A_{4} \beta^{4} e^{4 H}-A_{2} \beta^{2} e^{2 H}+1
$$

with entire $H, H(0)=0$, a constant $\beta \neq 0$ and

$$
A_{2}=4+2 u, \quad A_{4}=6+4 u+v^{2} .
$$

For $u, v$ we have

$$
v \neq 0, \quad u^{2} \neq v^{2}, \quad 16+8 u+v^{2} \neq 0 .
$$

4. In $\S 3$ we have gotten the representation of $g$ and hence the surface $R$ defined by $y^{2}=g(x)$. We shall now prove that this is really of maximal $B$ type. We may adopt

$$
g=-\frac{\left(a_{3}-a_{2}\right)^{2}\left(a_{1}-a_{2}\right)^{2}}{64 a_{3}^{2} \beta^{4} e^{4 H}} \prod_{\jmath=1}^{8}\left(\beta e^{H}-\lambda_{j}\right) .
$$


Let us consider the following function

$$
f_{1}+\sqrt{g}, \quad f_{1}^{2}=a_{1} a_{2}-g .
$$

This belongs to $\mathcal{E}(R)$. We now put

$$
f_{1}=\frac{1}{2 a_{3}}\left\{\frac{\left(a_{3}-a_{2}\right)\left(a_{1}-a_{2}\right)}{4}\left(\beta e^{H}-\frac{1}{\beta e^{H}}\right)^{2}-a_{1} a_{2}-a_{3}^{2}\right\} .
$$

This gives

$$
\begin{aligned}
& a_{3}^{2}-2 a_{3} f_{1}+a_{1} a_{2}=-\frac{\left(a_{3}-a_{2}\right)\left(a_{1}-a_{2}\right)}{4}\left(\beta e^{H}-\frac{1}{\beta e^{H}}\right)^{2} \equiv g_{3}^{2}, \\
& a_{4}^{2}-2 a_{4} f_{1}+a_{3} a_{4}=\frac{a_{1} a_{2}}{a_{3}^{2}}\left(a_{1} a_{2}-2 a_{3} f_{1}+a_{3}^{2}\right) \\
& =\frac{a_{1} a_{2}}{a_{3}^{2}} g_{3}^{2} \equiv g_{4}^{2}, \\
& a_{2}^{2}-2 a_{2} f_{1}+a_{1} a_{2} \\
& =a_{2}^{2}+\frac{a_{2}}{a_{3}}\left\{\frac{\left(a_{3}-a_{2}\right)\left(a_{1}-a_{2}\right)}{4}\left(\beta e^{H}-\frac{1}{\beta e^{H}}\right)^{2}-a_{3}^{2}-a_{1} a_{2}\right\}+a_{1} a_{2} \\
& =\frac{a_{2}}{4 a_{3}}\left(a_{3}-a_{2}\right)\left(a_{1}-a_{3}\right)\left(\beta e^{H}+\frac{1}{\beta e^{H}}\right)^{2} \equiv g_{2}^{2}, \\
& a_{1}^{2}-2 a_{1} f_{1}+a_{1} a_{2} \\
& =\frac{a_{3} a_{4}}{a_{2}^{2}}\left(a_{2}^{2}-2 a_{2} f_{1}+a_{1} a_{2}\right)=\frac{a_{3} a_{4}}{a_{2}^{2}} g_{2}^{2} \equiv g_{1}^{2} .
\end{aligned}
$$

Thus our $R$ belongs to the class of maximal $B$ type.

5. Let $S$ be another ultrahyperelliptic surface defined by $Y^{2}=G(X)$ with entire $G$ having only infinitely many simple zeros. Let $\phi$ be a non-trivial analytic mapping of $S$ into $R$. Then we have the following fact: If $R$ is of maximal $B$ type, then $S$ is also of maximal $B$ type if $\phi$ exists.

We shall prove this. Let $h$ be the projection of $\phi$, that is, $h=\mathscr{P}_{R^{\circ}} \phi \circ \mathcal{P}_{S}^{-1}$. Let $f$ be a member of $\mathcal{E}(R)$ such that $f$ satisfies two conditions of maximal $B$ type. Then

$$
\begin{aligned}
& f \circ \mathscr{D}_{R}^{-1}=f_{1}+f_{2} \sqrt{g}, \\
& a^{2}-2 a f_{1}+f_{1}^{2}-f_{2}^{2} g=c
\end{aligned}
$$

for some $a \neq \infty$ and for a non-zero constant c. Transplanting $f$ on $S$ by $\phi$, that is,

$$
f \circ \phi \circ \mathscr{P}_{S}^{-1}
$$

we have 


$$
\begin{aligned}
f \circ \phi \circ \mathscr{P}_{S}^{-1} & =f \circ \mathscr{P}_{R}^{-1} \circ \mathscr{P}_{R} \circ \phi \circ \mathscr{P}_{S}^{-1} \\
& =f \circ \mathscr{Q}_{R}^{-1} \circ h=f_{1} \circ h+f_{2} \circ h \sqrt{g \circ h} .
\end{aligned}
$$

Hence

$$
a^{2}-2 a f_{1} \circ h+\left(f_{1} \circ h\right)^{2}-\left(f_{2} \circ h\right)^{2} g \circ h=c .
$$

However by $[7]$

$$
f^{* 2} G=g \circ h
$$

with meromorphic $f^{*}$. However $g$ and $G$ have only simple zeros. Hence $f^{*}$ is entire. Thus

Let

$$
a^{2}-2 a f_{1} \circ h+\left(f_{1} \circ h\right)^{2}-\left(f_{2} \circ h\right)^{2} f^{* 2} G=c .
$$

$$
f \circ \mathscr{P}_{S}^{-1}=f_{1} \circ h+\left(f_{2} \circ h\right) f * \sqrt{G} .
$$

Then $\hat{f} \in \mathcal{E}(S)$ and $a$ is the desired lacunary value of $f$. The condition (2) in the definition of maximal $B$ type holds for $f$ with the same $a_{1}, a_{2}, a_{3}, a_{4}$. Thus we have the desired result.

6. Let $R$ be of maximal $B$ type. We shall consider the existence problem of analytic mappings of $R$ into another $S$ or of $S$ into $R$.

Assume that $P(S)=4$. Consider a non-trivial analytic mapping $\phi$ of $R$ into $S$. Then there exist an entire function $h$ and a meromorphic function $f$ such that

$$
\begin{aligned}
g & \equiv \beta^{8} e^{8 H}-A_{2} \beta^{6} e^{6 H}+A_{4} \beta^{4} e^{4 H}-A_{2} \beta^{2} e^{2 H}+1 \\
& =f(z)^{2}\left(e^{L_{\circ} h}-\delta_{1}\right)\left(e^{L \circ h}-\delta_{2}\right)
\end{aligned}
$$

with constants $\delta_{1}, \delta_{2}, \delta_{1} \delta_{2}\left(\delta_{1}-\delta_{2}\right) \neq 0$. For simplicity's sake we put $M=L \circ h-$ $L \circ h(0), c=\exp L \circ h(0)$. The right hand side is

$$
f(z)^{2}\left(c e^{M}-\delta_{1}\right)\left(c e^{M}-\delta_{2}\right) .
$$

Then

$$
\begin{aligned}
N_{2}(r, 0, g) & =N_{2}\left(r, 0,\left(c e^{M}-\delta_{1}\right)\left(c e^{M}-\delta_{2}\right)\right) \\
& \sim 2 m\left(r, e^{M}\right)
\end{aligned}
$$

and

$$
N_{2}(r, 0, g) \sim 8 m\left(r, e^{H}\right)
$$

with a negligible exceptional set of $r$. Hence

$$
4 m\left(r, e^{H}\right) \sim m\left(r, e^{M}\right) .
$$

Further

$$
2 N(r, 0, f) \leqq N_{1}(r, 0, g)+\bar{N}_{1}(r, 0, g)=o\left(m\left(r, e^{H}\right)\right)
$$




$$
\begin{aligned}
2 N(r, \infty, f) \leqq & \bar{N}_{1}\left(r, 0,\left(c e^{M}-\delta_{1}\right)\left(c e^{M}-\delta_{2}\right)\right) \\
& +\bar{N}_{1}\left(r, 0,\left(c e^{M}-\delta_{1}\right)\left(c e^{M}-\delta_{2}\right)\right) \\
= & o\left(m\left(r, e^{M}\right)\right) .
\end{aligned}
$$

By differentiation of

$$
g=f^{2}\left(c e^{M}-\delta_{1}\right)\left(c e^{M}-\delta_{2}\right)
$$

and by elimination of $f^{2}$ we have

$$
\begin{aligned}
& a_{1} e^{2 M+8 H}+a_{2} e^{2 M+6 H}+a_{3} e^{2 M+4 H}+a_{4} e^{2 M+2 H}+a_{5} e^{2 M} \\
& +a_{6} e^{M+8 H}+a_{7} e^{M+6 H}+a_{8} e^{M+4 H}+a_{9} e^{M+2 H}+a_{10} e^{M} \\
& +a_{11} e^{8 H}+a_{12} e^{6 H}+a_{13} e^{4 H}+a_{14} e^{2 H}+a_{15}=0, \\
& a_{1}=\left(\frac{2 f^{\prime}}{f}+2 M^{\prime}-8 H^{\prime}\right) \beta^{8} c^{2}, \quad a_{2}=\left(\frac{2 f^{\prime}}{f}+2 M^{\prime}-6 H^{\prime}\right)\left(-A_{2}\right) \beta^{6} c^{2}, \\
& a_{3}=\left(\frac{2 f^{\prime}}{f}+2 M^{\prime}-4 H^{\prime}\right) A_{4} \beta^{4} c^{2}, \quad a_{4}=\left(\frac{2 f^{\prime}}{f}+2 M^{\prime}-2 H^{\prime}\right)\left(-A_{2}\right) \beta^{2} c^{2}, \\
& a_{5}=\left(\frac{2 f^{\prime}}{f}+2 M^{\prime}\right) c^{2}, \quad a_{6}=\left(-\frac{2 f^{\prime}}{f}-M^{\prime}+8 H^{\prime}\right) \beta^{8}\left(\delta_{1}+\delta_{2}\right) c, \\
& a_{7}=\left(\frac{2 f^{\prime}}{f}+M^{\prime}-6 H^{\prime}\right) A_{2} \beta^{6}\left(\delta_{1}+\delta_{2}\right) c, \quad a_{8}=\left(-\frac{2 f^{\prime}}{f}-M^{\prime}+4 H^{\prime}\right) \beta^{4} A_{4}\left(\delta_{1}+\delta_{2}\right) c, \\
& a_{9}=\left(\frac{2 f^{\prime}}{f}+M^{\prime}-2 H^{\prime}\right) A_{2} \beta^{2}\left(\delta_{1}+\delta_{2}\right) c, \quad a_{10}=\left(-\frac{2 f^{\prime}}{f}-M^{\prime}\right)\left(\delta_{1}+\delta_{2}\right) c, \\
& a_{11}=\left(2 \frac{f^{\prime}}{f}-8 H^{\prime}\right) \beta^{8} \delta_{1} \delta_{2}, \quad a_{12}=\left(-\frac{2 f^{\prime}}{f}+6 H^{\prime}\right) A_{2} \beta^{6} \delta_{1} \delta_{2}, \\
& a_{13}=\left(2 \frac{f^{\prime}}{f}-4 H^{\prime}\right) A_{4} \beta^{4} \delta_{1} \delta_{2}, \quad a_{14}=\left(-\frac{2 f^{\prime}}{f}+2 H^{\prime}\right) A_{2} \beta^{2} \delta_{1} \delta_{2}, \\
& a_{15}=2 \frac{f^{\prime \prime}}{f} \delta_{1} \delta_{2} .
\end{aligned}
$$

Evidently $T\left(r, a_{j}\right)=N\left(r, \infty, a_{j}\right)+m\left(r, a_{j}\right)=o\left(m\left(r, e^{M}\right)\right)+o\left(m\left(r, e^{H}\right)\right)$ for every $j$, $1 \leqq j \leqq 15$. Now we can make use of Nevanlinna's proof [3] of the impossibility of Borel's identity. By $m\left(r, e^{4 H}\right) \sim m\left(r, e^{M}\right)$ we can save our consideration and conclude either $M=4 H$ or $M=-4 H$. Indeed we have firstly the existence of $\left(c_{j}\right)_{\jmath=1, \ldots, 14}$ such that

$$
\begin{aligned}
c_{1} a_{1} e^{2 M+6 H} & +c_{2} a_{2} e^{2 M+4 H}+c_{3} a_{3} e^{2 M+2 H}+c_{4} a_{4} e^{2 M} \\
& +c_{5} a_{5} e^{2 M-2 H}+c_{6} a_{6} e^{M+6 H}+c_{7} a_{7} e^{M+4 H}+c_{8} a_{8} e^{M+2 H} \\
& +c_{9} a_{9} e^{M}+c_{10} a_{10} e^{M-2 H}+c_{11} a_{11} e^{6 H}+c_{12} a_{12} e^{4 H}
\end{aligned}
$$




$$
+c_{13} a_{13} e^{2 H}+c_{14} a_{14}=0 \text {. }
$$

If $c_{i} c_{\jmath}=0(i \neq j, i, j=1, \cdots, 13)$, then we have only one possible case

$$
c_{7} a_{7} e^{M+4 H}+c_{14} a_{14}=0,
$$

which gives $M+4 H=0$. If there is at least one $c_{i} c_{j} \neq 0(i, j=1, \cdots, 13, i \neq j)$, then we have the existence of $\left(c_{j}^{\prime}\right)_{j=1, \ldots, 13}$ such that

$$
c_{1}^{\prime} a_{1} e^{2 M+4 H}+c_{2}^{\prime} a_{2} e^{2 M+2 H}+\cdots+c_{12}^{\prime} a_{12} e^{2 H}+c_{13}^{\prime} a_{13}=0 .
$$

If $c_{i}^{\prime} c_{j}^{\prime}=0(i \neq j, i, j=1, \cdots, 12)$, then we have two possible cases

and

$$
c_{6}^{\prime} a_{6} e^{M+4 H}+c_{13}^{\prime} a_{13}=0
$$

$$
c_{10}^{\prime} a_{10} e^{M-4 H}+c_{13}^{\prime} a_{13}=0 .
$$

These give either $M+4 H=0$ or $M-4 H=0$. If there is at least one $c_{i}^{\prime} c_{j}^{\prime} \neq 0(i \neq j$, $i, j=1, \cdots, 12$ ), we continue the same process repeatedly. In each step we have the desired result: $M=4 H$ or $M=-4 H$.

The case $M=4 H$. Then we have

$$
\begin{aligned}
a_{1} e^{16 H} & +a_{2} e^{14 H}+\left(a_{3}+a_{6}\right) e^{12 H}+\left(a_{4}+a_{7}\right) e^{10 H} \\
& +\left(a_{5}+a_{8}+a_{11}\right) e^{8 H}+\left(a_{9}+a_{12}\right) e^{6 H}+\left(a_{10}+a_{13}\right) e^{4 H} \\
& +a_{14} e^{2 H}+a_{15}=0 .
\end{aligned}
$$

By our earlier result in [2] this gives

$$
\begin{aligned}
a_{1} & =a_{2}=a_{3}+a_{6}=a_{4}+a_{7}=a_{5}+a_{8}+a_{11} \\
& =a_{9}+a_{12}=a_{10}+a_{13}=a_{14}=a_{15}=0 .
\end{aligned}
$$

Hence $f$ is a constant and $A_{2}=0$,

$$
c A_{4}=-\beta^{4}\left(\delta_{1}+\delta_{2}\right), \quad \beta^{8} \delta_{1} \delta_{2}=c^{2} .
$$

The case $M=-4 H$. Then we have

$$
\begin{aligned}
a_{5} e^{-8 H} & +a_{4} e^{-6 H}+\left(a_{3}+a_{10}\right) e^{-4 H}+\left(a_{2}+a_{9}\right) e^{-2 H} \\
& +a_{1}+a_{8}+a_{15}+\left(a_{7}+a_{14}\right) e^{2 H}+\left(a_{6}+a_{13}\right) e^{4 H} \\
& +a_{12} e^{6 H}+a_{11} e^{8 H}=0 .
\end{aligned}
$$

This gives

$$
\begin{aligned}
a_{5} & =a_{4}=a_{3}+a_{10}=a_{2}+a_{9}=a_{1}+a_{8}+a_{15} \\
& =a_{7}+a_{14}=a_{6}+a_{13}=a_{12}=a_{11}=0 .
\end{aligned}
$$

Hence 


$$
\begin{aligned}
& \frac{f^{\prime}}{f}=-M^{\prime}, \quad A_{2}=0, \\
& A_{4} \beta^{4} c=-\delta_{1}-\delta_{2}, \quad \beta^{8} c^{2}=\delta_{1} \delta_{2} .
\end{aligned}
$$

Hence we have the following

THEOREM 1. Let $R$ be of maximal $B$ type and let $S$ be the surface of $P(S)$ $=4$. Assume that there is a non-trivial analytic mapping $\phi$ of $R$ into $S$. Then, with entire projection $h$ of $\phi, A_{2}=0$ and either

or

$$
\begin{aligned}
& 4 H=L \circ h-L \circ h(0), \\
& A_{4}=-e^{-L \circ h(0)} \beta^{4}\left(\delta_{1}+\delta_{2}\right), \\
& e^{2 L \circ h(0)}=\beta^{8} \delta_{1} \delta_{2}
\end{aligned}
$$

$$
\begin{aligned}
& 4 H=-L \circ h+L \circ h(0), \\
& A_{4} \beta^{4}=-e^{-L_{\bullet} h(0)}\left(\delta_{1}+\delta_{2}\right), \\
& \beta^{8} e^{2 L \bullet h(0)}=\delta_{1} \delta_{2} .
\end{aligned}
$$

If the conditions hold, then $\phi$ exists.

The inverse statement is trivial by [7].

Corollary 1. Let $R$ be of maximal $B$ type. If $P(R)=4$, then $A_{2}=0$, that is, on assuming that 0 is lacunary

$$
2 a_{3}^{2}+a_{1} a_{2}+a_{3} a_{1}-a_{3} a_{2}+a_{2}^{2}=0
$$

and vice versa.

THEOREM 2. Let $R$ be of maximal $B$ type and let $S$ be the surface of $P(S)$ $=4$. Assume that there is a non-trivial analytic mapping $\phi$ of $S$ into $R$. Then $A_{2}=0$ and either

or

$$
\begin{aligned}
& 4 H \circ h-4 H \circ h(0)=L, \\
& A_{4}=-\beta^{4} e^{4 H \bullet h(0)}\left(\delta_{1}+\delta_{2}\right), \\
& \beta^{8} e^{8 H \bullet h(0)} \delta_{1} \delta_{2}=1
\end{aligned}
$$

$$
\begin{aligned}
& 4 H \circ h-4 H \circ h(0)=-L, \\
& A_{4} \beta^{4} e^{4 H \circ h(0)}=-\left(\delta_{1}+\delta_{2}\right), \\
& \beta^{8} e^{8 H \circ h(0)}=\delta_{1} \delta_{2} .
\end{aligned}
$$

If the conditions hold, then $\phi$ exists. 
There is an ultrahyperelliptic surface $R$ of maximal $B$ type and with $P(R)$ $=3$. It is known that $P(R) \geqq 3$ implies

$$
g=1-2 \beta_{1} e^{H}-2 \beta_{2} e^{L}+\beta_{1}^{2} e^{2 H}-2 \beta_{1} \beta_{2} e^{H+L}+\beta_{2}^{2} e^{2 L}
$$

with two entire functions $H, L(H(0)=L(0)=0)$ and non-zero constants $\beta_{1}, \beta_{2}$. Let us put $2 H=L$. Then we have

$$
g=1-2 \beta_{1} e^{H}+\left(\beta_{1}^{2}-2 \beta_{2}\right) e^{2 H}-2 \beta_{1} \beta_{2} e^{3 H}+\beta_{2}^{2} e^{4 H} .
$$

If we put

$$
\begin{aligned}
& -2 \beta_{1}=-A_{2} \beta^{2}, \\
& \beta_{1}^{2}-2 \beta_{2}=A_{4} \beta^{4}, \\
& 2 \beta_{1} \beta_{2}=A_{2} \beta^{6}, \\
& \beta_{2}^{2}=\beta^{8},
\end{aligned}
$$

then $g$ has the form of maximal $B$ type. In this case

$$
\beta^{4}=\beta_{2}, \quad 4 \beta_{1}^{2}=A_{2}^{2} \beta_{2}, \quad A_{2}^{2}=4 A_{4}+8 .
$$

Hence $a_{1}, a_{2}, a_{3}, a_{4}$ must satisfy

$$
a_{1} a_{2}=a_{3} a_{4}, \quad 16 a_{1} a_{2} a_{3}^{2}=\left(a_{3}-a_{2}\right)^{2}\left(a_{1}-a_{2}\right)^{2} .
$$

Next we shall prove that

$$
y^{2}=1-2 \beta_{1} e^{H}+\left(\beta_{1}^{2}-2 \beta_{2}\right) e^{2 H}-2 \beta_{1} \beta_{2} e^{3 H}+\beta_{2}^{2} e^{4 H} \equiv g_{1}
$$

determine a surface of $P(S)=3$, when $16 \beta_{2} \neq \beta_{1}^{2}$.

If $16 \beta_{2} \neq \beta_{1}^{2}$, it is easy to prove

$$
N_{2}\left(r, 0, g_{1}\right) \sim 4 m\left(r, e^{H}\right) .
$$

Assume that $P(S)=4$. Then

$$
g_{1}=f^{2}\left(e^{L}-\delta_{1}\right)\left(e^{L}-\delta_{2}\right), \quad \delta_{1} \delta_{2}\left(\delta_{1}-\delta_{2}\right) \neq 0 .
$$

Then the similar consideration as in the proof of Theorem 1 does work. And we have either $L=2 H$ or $L=-2 H$. If $L=2 H$, then $a_{15}=0$ implies the constancy of $f$. Thus

$$
\begin{gathered}
1-2 \beta_{1} e^{H}+\left(\beta_{1}^{2}-2 \beta_{2}\right) e^{2 H}-2 \beta_{1} \beta_{2} e^{3 H}+\beta_{2}^{2} e^{4 H} \\
=c^{2}\left(e^{4 H}-\left(\delta_{1}+\delta_{2}\right) e^{2 H}+\delta_{1} \delta_{2}\right) .
\end{gathered}
$$

This gives $\beta_{1}=0$, which is a contradiction. If $L=-2 H$, then $a_{5}=a_{11}=0$. Hence we have

$$
\frac{f^{\prime}}{f}=4 H^{\prime}, \quad f=f(0) e^{4 H}
$$


Thus

$$
\begin{gathered}
1-2 \beta_{1} e^{H}+\left(\beta_{1}^{2}-2 \beta_{2}\right) e^{2 H}-2 \beta_{1} \beta_{2} e^{3 H}+\beta_{2}^{2} e^{4 H} \\
=c^{2} e^{8 H}\left(e^{-4 H}-\left(\delta_{1}+\delta_{2}\right) e^{-2 H}+\delta_{1} \delta_{2}\right) .
\end{gathered}
$$

This gives $\beta_{1}=0$, which is a contradiction. Therefore $P(S)=3$.

Assume that $16 \beta_{2}=\beta_{1}^{2}$. Then $N_{2}\left(r, 0, g_{1}\right) \sim 2 m\left(r, e^{H}\right)$. However $N_{2}(r, 0, g) \sim$ $4 m\left(r, e^{H}\right)$ if

$$
g=1-A_{2} \beta^{2} e^{H}+A_{4} \beta^{4} e^{2 H}-A_{2} \beta^{6} e^{3 H}+\beta^{8} e^{4 H}
$$

with $\beta^{4}=\beta_{2}, 4 \beta_{1}^{2}=A_{2}^{2} \beta_{2}, A_{2}^{2}=4 A_{4}+8$. This is a contradiction. Therefore $16 \beta_{2} \neq \beta_{1}^{2}$. Thus $P(R)=3$.

7. Let $R$ and $S$ be of maximal $B$ type. Let $\phi$ be a non-trivial analytic mapping of $R$ into $S$. Then

$$
\begin{aligned}
g & \equiv \beta^{4} e^{4 H}-A_{2} \beta^{3} e^{3 H}+A_{4} \beta^{2} e^{2 H}-A_{2} \beta e^{H}+1 \\
& =f^{2}\left[\gamma^{4} e^{4 L_{\circ} h}-B_{2} \gamma^{3} e^{3 L_{\circ} h}+B_{4} \gamma^{2} e^{2 L_{\circ} h}-B_{2} \gamma e^{L_{\circ} h}+1\right] \\
& \equiv f^{2} G \circ h .
\end{aligned}
$$

Let $L \circ h-L \circ h(0)$ be $M$ and let $c$ be $\exp L \circ h(0)$. Then

$$
g=f^{2}\left[\gamma^{4} c^{4} e^{4 M}-B_{2} \gamma^{3} c^{3} e^{3 M}+B_{4} \gamma^{2} c^{2} e^{2 M}-B_{2} \gamma c e^{M}+1\right] .
$$

By differentiation of this equation and by elimination of $f^{2}$ we have

$$
\begin{gathered}
a_{1} e^{4 H+4 M}+a_{2} e^{3 H+4 M}+a_{3} e^{2 H+4 M}+a_{4} e^{H+4 M}+a_{5} e^{4 M} \\
+a_{6} e^{4 H+3 M}+a_{7} e^{3 H+3 M}+a_{8} e^{2 H+3 M}+a_{9} e^{H+3 M}+a_{10} e^{3 M} \\
\quad+a_{11} e^{4 H+2 M}+a_{12} e^{3 H+2 M}+a_{13} e^{2 H+2 M}+a_{14} e^{H+2 M}+a_{15} e^{2 M} \\
\quad+a_{16} e^{4 H+M}+a_{17} e^{3 H+M}+a_{18} e^{2 H+M}+a_{19} e^{H+M}+a_{20} e^{M} \\
\quad+a_{21} e^{4 H}+a_{22} e^{3 H}+a_{23} e^{2 H}+a_{24} e^{H}+a_{25}=0, \\
a_{1}=\left(4 H^{\prime}-\frac{2 f^{\prime}}{f}-4 M^{\prime}\right) \beta^{4} \gamma^{4} c^{4}, \quad a_{2}=A_{2}\left(-3 H^{\prime}+\frac{2 f^{\prime}}{f}+4 M^{\prime}\right) \beta^{3} \gamma^{4} c^{4}, \\
a_{3}=A_{4}\left(2 H^{\prime}-\frac{2 f^{\prime}}{f}-4 M^{\prime}\right) \beta^{2} \gamma^{4} c^{4}, \quad a_{4}=A_{2}\left(-H^{\prime}+\frac{2 f^{\prime}}{f}+4 M^{\prime}\right) \beta \gamma^{4} c^{4}, \\
a_{5}=\left(-\frac{2 f^{\prime}}{f}-4 M^{\prime}\right) \gamma^{4} c^{4}, \quad a_{6}=-B_{2}\left(4 H^{\prime}-\frac{2 f^{\prime}}{f}-3 M^{\prime}\right) \beta^{4} \gamma^{3} c^{3}, \\
a_{7}=-B_{2} A_{2}\left(-3 H^{\prime}+\frac{2 f^{\prime}}{f}+3 M^{\prime}\right) \beta^{3} \gamma^{3} c^{3}, \quad a_{8}=-B_{2} A_{4}\left(2 H^{\prime}-\frac{2 f^{\prime}}{f}-3 M^{\prime}\right) \beta^{2} \gamma^{3} c^{3},
\end{gathered}
$$




$$
\begin{aligned}
& a_{9}=-B_{2} A_{2}\left(-H^{\prime}+\frac{2 f^{\prime}}{f}+3 M^{\prime}\right) \beta \gamma^{3} c^{3}, \quad a_{10}=-B_{2}\left(-\frac{2 f^{\prime}}{f}-3 M^{\prime}\right) \gamma^{3} c^{3}, \\
& a_{11}=B_{4}\left(4 H^{\prime}-\frac{2 f^{\prime}}{f}-2 M^{\prime}\right) \beta^{4} \gamma^{2} c^{2}, \quad a_{12}=B_{4} A_{2}\left(-3 H^{\prime}+\frac{2 f^{\prime}}{f}+2 M^{\prime}\right) \beta^{3} \gamma^{2} c^{2}, \\
& a_{13}=B_{4} A_{4}\left(2 H^{\prime}-\frac{2 f^{\prime}}{f}-2 M^{\prime}\right) \beta^{2} \gamma^{2} c^{2}, \quad a_{14}=B_{4} A_{2}\left(-H^{\prime}+\frac{2 f^{\prime}}{f}+2 M^{\prime}\right) \beta \gamma^{2} c^{2}, \\
& a_{15}=B_{4}\left(-\frac{2 f^{\prime}}{f}-2 M^{\prime}\right) \gamma^{2} c^{2}, \quad a_{16}=-B_{2}\left(4 H^{\prime}-\frac{2 f^{\prime}}{f}-M^{\prime}\right) \beta^{4} \gamma c, \\
& a_{17}=B_{2} A_{2}\left(3 H^{\prime}-\frac{2 f^{\prime}}{f}-M^{\prime}\right) \beta^{3} \gamma c, \quad a_{18}=-B_{2} A_{4}\left(2 H^{\prime}-\frac{2 f^{\prime}}{f}-M^{\prime}\right) \beta^{2} \gamma c, \\
& a_{19}=B_{2} A_{2}\left(H^{\prime}-\frac{2 f^{\prime}}{f}-M^{\prime}\right) \beta \gamma c, \quad a_{20}=B_{2}\left(\frac{2 f^{\prime}}{f}+M^{\prime}\right) \gamma c, \\
& a_{21}=\left(4 H^{\prime}-\frac{2 f^{\prime}}{f}\right) \beta^{4}, \quad a_{22}=-A_{2}\left(3 H^{\prime}-\frac{i 2 f^{\prime}}{f}\right) \beta^{3}, \\
& a_{23}=A_{4}\left(2 H^{\prime}-\frac{2 f^{\prime}}{f}\right) \beta^{2}, \quad a_{24}=-A_{2}\left(H^{\prime}-\frac{2 f^{\prime}}{f}\right) \beta, \quad a_{25}=-\frac{2 f^{\prime}}{f} .
\end{aligned}
$$

In the present case we have

$$
4 m\left(r, e^{H}\right) \sim N_{2}(r, 0, g)=N_{2}(r, 0, G \circ h) \sim 4 m\left(r, e^{M}\right)
$$

and

$$
N_{1}(r, \infty, f)=o\left(m\left(r, e^{H}\right)\right)
$$

Hence

$$
T\left(r, a_{j}\right)=o\left(m\left(r, e^{H}\right)\right) .
$$

Thus we can make use of Nevanlinna's method of proof of the impossibility of Borel's identity. In our case $m\left(r, e^{H}\right) \sim m\left(r, e^{M}\right)$ brings us a simplicity. By a similar consideration as in $\S 6$ we only have two possibilities: a) $H=M$ or b) $H=-M$.

Case a). We have

$$
\begin{aligned}
a_{2} e^{8 H} & +\left(a_{2}+a_{6}\right) e^{7 H}+\left(a_{3}+a_{7}+a_{11}\right) e^{6 H}+\left(a_{4}+a_{8}+a_{12}+a_{16}\right) e^{5 H} \\
& +\left(a_{5}+a_{9}+a_{13} a+a_{17}+a_{21}\right) e^{4 H}+\left(a_{10}+a_{14}+a_{18}+a_{22}\right) e^{3 H} \\
& +\left(a_{15}+a_{19}+a_{23}\right) e^{2 H}+\left(a_{20}+a_{24}\right) e^{H}+a_{25}=0 .
\end{aligned}
$$

Hence $a_{25}=0$ and hence $f$ is a constant. Therefore

$$
\begin{aligned}
& \beta^{4}=f^{2} \gamma^{4} c^{4}, \quad A_{2} \beta^{2}=f^{2} B_{2} \gamma^{3} c^{3}, \quad A_{4} \beta^{2}=B_{4} \gamma^{2} c^{2} f^{2}, \\
& A_{2} \beta=f^{2} \gamma c B_{2}, \quad f^{2}=1 .
\end{aligned}
$$


These give $\beta^{4}=\gamma^{4} c^{4}$. If $\beta^{2}=\gamma^{2} c^{2}$, we have $A_{4}=B_{4}, A_{2}=B_{2}$ or $A_{2}=-B_{2}$. If $\beta^{2}$ $=-\gamma^{2} c^{2}$, we have $B_{2}=A_{2}=0$ and $A_{4}=-B_{4}$.

Case b). We have

$$
\begin{aligned}
a_{21} e^{8 H} & +\left(a_{16}+a_{22}\right) e^{7 H}+\left(a_{11}+a_{17}+a_{23}\right) e^{6 H}+\left(a_{6}+a_{12}+a_{18}+a_{24}\right) e^{5 H} \\
& +\left(a_{1}+a_{7}+a_{13}+a_{19}+a_{25}\right) e^{4 H}+\left(a_{2}+a_{8}+a_{14}+a_{20}\right) e^{3 H} \\
& +\left(a_{3}+a_{9}+a_{15}\right) e^{2 H}+\left(a_{4}+a_{10}\right) e^{H}+a_{5}=0 .
\end{aligned}
$$

Hence $a_{21}=0$ and $f=f(0) e^{2 H}$. Thus

$$
\begin{aligned}
& \beta^{4} e^{4 H}-A_{2} \beta^{3} e^{3 H}+A_{4} \beta^{2} e^{2 H}-A_{2} \beta e^{H}+1 \\
& =d^{2}\left[\gamma^{4} c^{4}-B_{2} \gamma^{3} c^{3} e^{H}+B_{4} \gamma^{2} c^{2} e^{2 H}-B_{2} \gamma c e^{3 H}+e^{4 H}\right] .
\end{aligned}
$$

Therefore

$$
\begin{aligned}
& \beta^{4}=d^{2}, \quad A_{2} \beta^{3}=B_{2} \gamma c d^{2}, \quad A_{4} \beta^{2}=B_{4} d^{2} \gamma^{2} c^{2}, \\
& A_{2} \beta=B_{2} \gamma^{3} c^{3} d^{2}, \quad 1=d^{2} \gamma^{4} c^{4} .
\end{aligned}
$$

Hence

$$
A_{2} \beta^{3} \gamma^{3} c^{3}=B_{2}, \quad A_{2} \beta \gamma c=B_{2}, \quad \beta^{4} \gamma^{4} c^{4}=1 .
$$

If $\beta \gamma c=1$, then $A_{2}=B_{2}$ and $A_{4}=B_{4}$. If $\beta \gamma c=-1$, then $A_{2}=-B_{2}$ and $A_{4}=B_{4}$. If $\beta \gamma c=i$, then $A_{2}=B_{2}=0$ and $A_{4}=-B_{4}$. If $\beta \gamma c=-i$, then $A_{2}=B_{2}=0$ and $A_{4}=$ $-B_{4}$. Therefore we have the following

Theorem 3. Let $R$ and $S$ be of maximal $B$ type. Assume that there is a nontrivial analytic mapping $\phi$ of $R$ into $S$. Then there exists an entire function $h$ such that either $H=L \circ h-L \circ h(0)$ and one of the following four holds.

$$
\begin{aligned}
& \left\{\begin{array} { l } 
{ \beta = \gamma \operatorname { e x p } ( L \circ h ( 0 ) ) } \\
{ A _ { 4 } = B _ { 4 } } \\
{ A _ { 2 } = B _ { 2 } , }
\end{array} \quad \left\{\begin{array}{l}
\beta=-\gamma \exp (L \circ h(0)) \\
A_{4}=B_{4} \\
A_{2}=-B_{2},
\end{array}\right.\right. \\
& \left\{\begin{array} { l } 
{ \beta = i \gamma \operatorname { e x p } ( L \circ h ( 0 ) ) } \\
{ A _ { 4 } = - B _ { 4 } } \\
{ A _ { 2 } = B _ { 2 } = 0 , }
\end{array} \quad \left\{\begin{array}{l}
\beta=-\imath \gamma \exp (L \circ h(0)) \\
A_{4}=-B_{4} \\
A_{2}=B_{2}=0,
\end{array}\right.\right.
\end{aligned}
$$

or $H=-L \circ h+L \circ h(0)$ and one of the following four holds:

$$
\left\{\begin{array} { l } 
{ \beta \gamma \operatorname { e x p } ( L \circ h ( 0 ) ) = 1 } \\
{ A _ { 4 } = B _ { 4 } } \\
{ A _ { 2 } = B _ { 2 } , }
\end{array} \quad \left\{\begin{array}{l}
\beta \gamma \exp (L \circ h(0))=-1 \\
A_{4}=B_{4} \\
A_{2}=-B_{2},
\end{array}\right.\right.
$$




$$
\left\{\begin{array} { l } 
{ \beta \gamma \operatorname { e x p } ( L \circ h ( 0 ) ) = \imath } \\
{ A _ { 4 } = - B _ { 4 } } \\
{ A _ { 2 } = B _ { 2 } = 0 , }
\end{array} \quad \left\{\begin{array}{l}
\beta \gamma \exp (L \circ h(0))=-i \\
A_{4}=-B_{4} \\
A_{2}=B_{2}=0 .
\end{array}\right.\right.
$$

The inverse statement is also true.

8. We here mention some remarks.

We can prove that the following types do not occur: The condition (1) holds and in the condition (2)

$$
\left(n_{1}, n_{2}, n_{3}\right)=(2,3,5),(2,3,4),(2,3,3),(2,2,3)
$$

instead of

$$
\sum_{\jmath=1}^{t}\left(1-\frac{1}{n_{\jmath}}\right)=2 .
$$

We can also prove that, if the condition (1) holds,

$$
\left(n_{1}, n_{2}, n_{3}\right)=(2,2,2)
$$

implies the existence of another value $a_{4}$ defined by $a_{1} a_{2}=a_{3} a_{4}$, when $a=0$, say, and the function $g_{4}$ as in our result mentioned already, that is, $R$ belongs to the class of maximal $B$ type.

\section{REFERENCES}

[1] Hiromi, G. And H. Mutō, On the existence of analytic mappings, I. Kōdai Math. Sem. Rep. 19 (1967), 236-244.

[2] Hiromi, G. AND M. Ozawa, On the existence of analytic mappings between two ultrahyperelliptic surfaces. Kōdai Math. Sem. Rep. 17 (1965), 281-306.

[3] Nevanlinna, R., Einige Eindeutigkeitssätzes in der Theorie der meromorphen Funktionen. Acta Math. 48 (1926), 367-391.

[4] Ozawa, M., On complex analytic mappings. Kōdai Math. Sem. Rep. 17 (1965), 93-102.

[5] Ozawa, M., On ultrahyperelliptic surfaces. Kōdai Math. Sem. Rep. 17 (1965), 103-108.

[6] Ozawa, M., On complex analytic mappings between two ultrahyperelliptic surfaces. Kōdai Math. Sem. Rep. 17 (1965), 158-165.

[7] Ozawa, M., On the existence of analytic mappings. Kōdai Math. Sem. Rep. 17 (1965), 191-197.

[8] Ozawa, M., On an ultrahyperelliptic surface whose Picard's constant is three. Kōdai Math. Sem. Rep. 19 (1967), 245-256.

[9] OzAwa, M., A remark on ultrahyperelliptic surfaces. Kōdai Math. Sem. Rep. 19 (1967), 381-383.

[10] Ozawa, M. And N. Suita, On the existence of analytic mappings. Kōdai Math. Sem. Rep. 25 (1973), 397-405. 
[11] SelBerg, H.L., Algebroide Funktionen und Umkehrfunktionen Abelscher Integrale. Avh. Norske Vid. Akad. Oslo 8 (1934), 1-72.

[12] TodA, N., On the functional equation $\sum_{\imath=0}^{p} a_{\imath} f_{\imath}^{n_{i}}=1$. Tôhoku Journ. Math. 23 (1971), 289-299.

Department of Mathematics

Tokyo Institute of Technology

Oh-OKayama, Meguro-Ku, TOKyo

JAPAN 
